Y. Mewald

G. F. Hamann

\title{
Versagen des zentralen, peripheren und vegetativen Nervensystems
}

are caused by toxic-metabolic encephalopathy due to sepsis or liver or renal insufficiency, cerebrovascular disorders due to disseminated intravascular coagulation or other states with increased coagulation activity, by intracranial bleedings due to secondary bleeding disorders or reduced nutritive compensation (e.g. disorders of water and electrolyte balance). Complications of the peripheral nervous system include singular or multiple compression syndromes of peripheral nerves or critical illness polyneuropathy. The clinical symptoms include weaning difficulties and distal symmetrical paresis. The pathophysiology is complex and yet not well understood. The critical illness myopathy is often seen after concomittant use of corticosteroids. The autonomic nervous system is regularly involved in intensive care patients. Acute severe sympathetic hyperactivation might result in accompanying parasympathetic activation with danger of severe cardiac arrhythmia like the "torsade de points". Further autonomic disorders include electrolyte changes (e.g. the SIADH), metabolic disorders and fever. Treatment of all neurological complications is based on symptom control and the treatment of the underlying disease.

\section{Key words}

Septic encephalopathy - coma critical illness polyneuropathy critical illness myopathy vegetative crisis

Zusammenfassung Neurologische Komplikationen nach Sepsis oder Multiorganversagen sind häufig und wesentliche prognostische Faktoren eines Intensivpatienten. Im Wesentlichen können Störungen des zentralen, peripheren und vegetativen Nervensystems und der Muskulatur unterschieden werden. Störungen des zentralen Nervensystems mit den klinischen Leitsymptomen der Bewusstseinsstörung (Somnolenz bis Koma) oder Bewusstseinsveränderung (Verwirrtheit) und zentralen Lähmungen (Halbseitensyndrom) umfassen die toxische Enzephalopathie (z. B. im Rahmen einer Sepsis oder eines Leber- oder Nierenversagens), zerebrovaskuläre Komplikationen durch disseminierte intravasale Gerinnung oder Neigung zu thromboembolischen Komplikationen sowie intrakranielle Blutungen bei Gerinnungsstörungen oder nutritiv-toxisch bedingte Mangelzustände (z. B. bei Störungen des Wasser- und Elektrolythaushaltes). Die Hauptkomplikationen des peripheren Nervensystems umfassen lagerungsbedingte Schäden einzelner oder 
mehrerer peripherer Nerven und die sog. Intensivpolyneuropathie oder CIP (critical illness polyneuropathy). Häufigste Symptome der CIP sind die verzögerte Entwöhnung vom Respirator und distale, symmetrische und schlaffe Paresen. Die Pathophysiologie ist komplex und bis heute nur unvollständig verstanden. Die Intensivmyopathie oder CIM (critical illness myopathy) ist v.a. bei zu- sätzlicher Anwendung von Kortikosteroiden häufiger. Vegetative Störungen gehören regelhaft $\mathrm{zu}$ intensivmedizinischen Erkrankungen. Im Rahmen von sympathikotonen Reaktionen können durch parasympathische Gegenregulationen schwere Herzrhythmusstörungen bis hin zur "torsade de points" neben Elektrolytstörungen (z. B. SIADH), metabolischen Entgleisungen und Tempe- raturragulationsstörungen ausgelöst werden. Die jeweilige Therapie ist in der Regel symptomatisch und an der Behandlung der Grundkrankheit orientiert.

\section{Schlüsselwörter}

Septische Enzephalopathie Koma -

Critical Illness Polyneuropathie Criticall Illness Myopathie -

Vegetative Krisen

\section{Einleitung}

Viele Intensivpatienten entwickeln primär oder im Laufe ihres Intensivaufenthaltes eine Sepsis und/oder ein Multiorganversagen. Diese Komplikationen oder Ursachen der Intensivbehandlung verursachen erheblich längere Intensivaufenthalte, erhöhen dementsprechend die Kosten und verschlechtern entscheidend die Prognose der betroffenen Patienten. Zusätzlich kommt es $\mathrm{zu}$ neurologischen Komplikationen, die wiederum die Schwere des Krankheitsbildes verstärken, sekundäre Komplikationen (wie z.B. verzögerte Entwöhnung vom Respirator) und eine prognostische Verschlechterung für den Patienten bewirken. Durch eine zunehmende Verbesserung der intensivmedizinischen Behandlungsmöglichkeiten und verfügbarer Techniken rücken die neurologischen Komplikationen in den letzten Jahren vermehrt in den Blickpunkt des Interesses. Dies nicht zuletzt dadurch, dass viele Intensivpatienten überleben und diese Komplikationen erfahren, die früher an anderen Problemen verstorben wären.

Die Häufigkeit von neurologischen Komplikationen bei Intensivpatienten verschiedenster Fachrichtungen (meist internistisch oder chirurgisch) schwankt beträchtlich, man kann von ca. 30\% als niedrigstem und $90 \%$ als höchstem Wert ausgehen. Die klinische Praxis zeigt, dass fast jeder Intensivpatient mit einer längeren Intensivtherapie (über drei Wochen) zumindest eine neurologische Komplikation entwickelt. Zur Erkennung und Behandlung der neurologischen Komplikationen bedarf es einer guten und engen interdisziplinären Zusammenarbeit und gegenseitiger Beachtung von Therapieregimen und Leitlinien.

Im Folgenden werden die wesentlichen Erkrankungen vorgestellt:

\section{Septische Enzephalopathie}

\section{Definition}

Die septische Enzephalopathie ist eine akut auftretende diffuse zerebrale Funktionsstörung, die durch Veränderung in der Vigilanz in Erscheinung tritt. Sie ist Folge einer systemischen Infektion, ohne dass dabei eine direkte Infektion oder sonstige metabolisch bzw. medikamentös bedingte Beeinträchtigungen des Gehirns selbst vorliegen.

\section{Epidemiologie}

Es kann keine genaue Aussage über die Häufigkeit der septischen Enzephalopathie gemacht werden. In der Literatur findet man Angaben zwischen 9-80\% $[8,26,34]$, je nach Auswahl der Diagnosekriterien des jeweiligen Autors. In einer Studie mit $1333 \mathrm{~Pa}-$ tienten fanden Sprung et al. bei 23\% der Patienten einen direkten Zusammenhang zwischen dem Auftreten von Veränderungen des mentalen Status und dem Beginn einer Sepsis.

\section{Symptomatik}

Die septische Enzephalopathie manifestiert sich häufig und oft sehr früh im Verlauf der Sepsis. Dabei geht sie internistischen Leitsymptomen und Organmanifestationen sogar meist voraus. Leitsymptom ist die beeinträchtigte Vigilanz, die je nach Schwere der Sepsis von leichten Bewusstseinsstörungen bis hin zum Koma reichen kann. Typisch ist eine fluktuierende, anfänglich unspezifische Symptomatik (inadäquates Verhalten), die nur selten mit fokalneurologischen Defiziten einhergeht $[2,35]$.

Die Bandbreite an möglichen Symptomen ist groß. Milde Formen äußern sich in Teilnahmslosigkeit, inadäquaten Reaktionen, mnestischen Störun- 
Tab. 1 Schweregrad der septischen Enzephalopathie

\begin{tabular}{ll}
\hline Schweregrad & Symptome \\
\hline I & $\begin{array}{l}\text { Nachlassende Gedächtnisleistung, Konzentrations- und } \\
\text { Wahrnehmungsstörungen, Schreibstörungen }\end{array}$ \\
II & Somnolenz, Verwirrtheit, räumliche und zeitliche \\
& Desorientiertheit \\
III & Muskelrigidität oder Stupor, Krampfanfälle, \\
IV & Blicklähmungen, Hemiparesen \\
& Koma \\
\hline
\end{tabular}

Nach Bolanos et al. 1997 (aus Eggers et al. 2003 Anaesthesist 52:294-303)

gen, Konzentrationsstörungen oder psychomotorischer Unruhe. Schwere Formen sind gekennzeichnet durch Delirium, Sopor oder Koma. Bei der Untersuchung fällt in zirka $30 \%$ eine Rigidität auf [26] und nicht selten erleiden die Patienten epileptische Anfälle [19]. Weitere Symptome sind Irritiertheit, Verwirrtheit, Desorientiertheit, Antriebsstörungen, Beeinträchtigung der Aufmerksamkeit und des Schreibens, Halluzinationen und Verkennungen, also Symptome eines Delirs. Tremor oder multifokale Myokloni lassen sich eher selten feststellen $[4,26]$. In einer Studie von Wijdicks et al. mit 84 Patienten mit Sepsis und Multiorganversagen zeigte sich ein signifikanter Zusammenhang zwischen schwerer Hypotension und der Entwicklung einer septischen Enzephalopathie [41]. Eine Graduierung der septischen Enzephalopathie wurde vorgeschlagen, die praktikabel ist und eine prognostische Relevanz hat (Tab. 1).

\section{Pathophysiologie}

Die Pathophysiologie der septischen Enzephalopathie ist bis heute nicht vollständig erklärt. Es werden viele Faktoren diskutiert, die bei der Entstehung eine Rolle spielen können. Wesentlich beteiligt scheinen die durch Endotoxine getriggert aus Makrophagen und Lymphozyten ausgeschütteten Zytokine und andere Mediatoren zu sein. Diese können direkt oder über Radikalbildung zytotoxisch wirken und verschiedene Abläufe in Gang setzen, die zu metabolischen Störungen sowie Veränderungen im zerebralen Aminosäuren-, Glukose- und Neurotransmitterstoffwechsel führen. Hohe Zytokinspiegel verursachen Endothelschäden mit resultierender erhöhter Kapillarpermeabilität und zerstören damit die Bluthirnschranke (BHS). In der Folge kommt es $\mathrm{zu}$ einem Gewebsödem, Einblutungen und Neuronenuntergängen [2].

Zytokine führen über eine Induktion von Stickstoffmonoxid produzierenden Enzymen zu einer Vasodilatation mit Abnahme des systolischen Blut- drucks [2, 29]. Dies hat eine Störung der Makround Mikrozirkulation zur Folge, die zusammen mit einem Ausfall der metabolisch adaptierten Perfusionssteuerung eine homogene druckpassive Perfusionsverteilung und damit häufig eine verminderte zerebrale Perfusion nach sich zieht $[2,20]$. Es kommt zur hypoxischen Gewebsschädigung.

Die Aktivierung des Komplementsystems und der Gerinnung durch Endotoxine bedingt zusätzliche zerebrale Läsionen.

Eine weitere Theorie beruht auf den Auswirkungen eines beobachteten Missverhältnisses von verzweigtkettigen und aromatischen Aminosäuren (AS) $[11,12,19]$ bei gestörter Leberfunktion zugunsten der aromatischen AS. In Tierversuchen konnte gezeigt werden, dass es in den ersten Stunden der Sepsis zu einer Störung der BHS mit einer Beeinträchti-

\section{PATHOPHYSIOLOGIE DER ENZEPHALOPATHIE NACH SEPSIS UND MULTIORGANVERSAGEN}

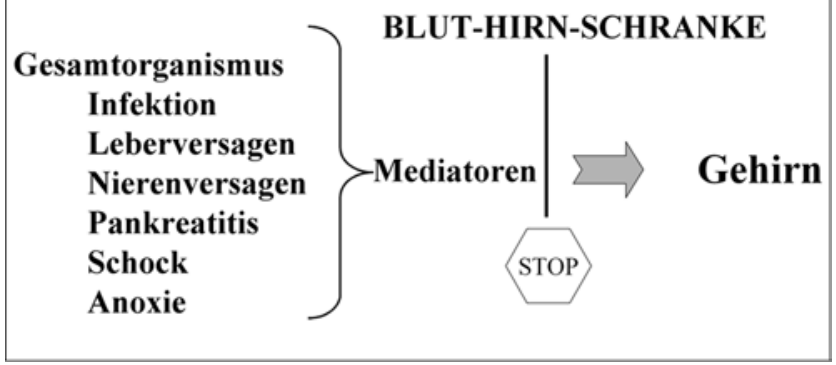

Abb. 1 Pathophysiologie der Enzephalopathie nach Sepsis und Multiorganversagen

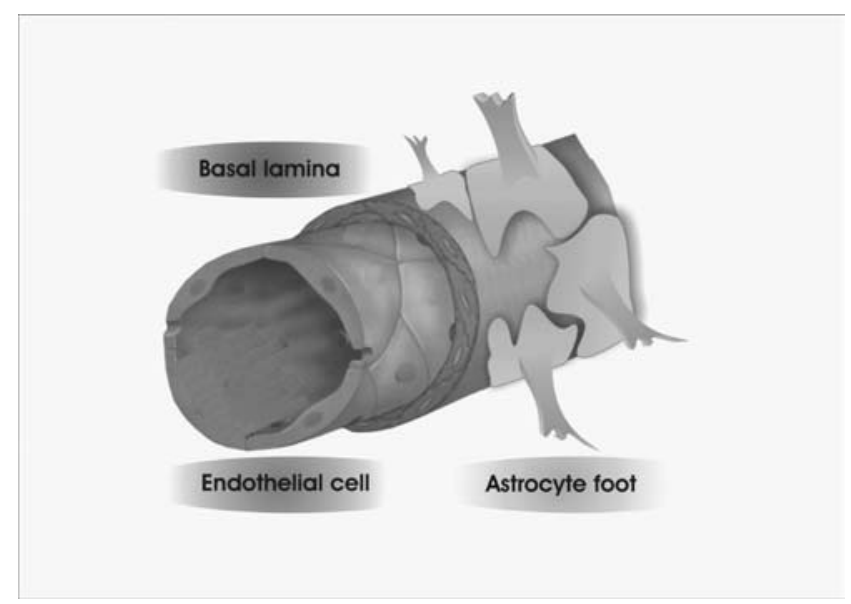

Abb. 2 Schema eines zerebralen Mikrogefäßes. Hervorzuheben ist, dass die Endothelzellen über besonders dichte tight junctions verbunden sind (BHS) und dass als äußere Umscheidung Astrozytenfortsätze um die Gefäße herumliegen 
gung des AS-Transportes über die BHS kommt. Die aromatischen AS (aus Muskelproteolyse und Katabolismus) werden schneller und vermehrt ins ZNS aufgenommen und führen dort zu einem gestörten Neurotransmitterprofil mit Bildung falscher Neurotransmitter [10-12].

Überhaupt scheint neben den oben erwähnten inflammatorischen Mechanismen noch eine begleitende BHS-Störung entscheidend $\mathrm{zu}$ sein, damit es überhaupt zu einer Exposition des Gehirns mit den inflammatorischen Mediatoren kommt. Somit ist die Störung der BHS eine wesentliche pathophysiologische Grundlage der Entwicklung einer septischen Enzephalopathie (Abb. 1 und 2).

Zusätzlich wird der Einfluss von reduzierter zerebraler Durchblutung und intrakranieller Drucksteigerung diskutiert [8].

\section{Diagnostik}

EEG: Nach einer Studie von Young et al. mit 62 septischen Patienten ist das EEG der sensitivste Parameter in der Diagnostik der septischen Enzephalopathie. Es zeigen sich unspezifische reversible Allgemeinveränderungen, die mit dem Schweregrad der Sepsis zunehmen. $\mathrm{Zu}$ diesen zählen eine Grundrhythmusverlangsamung mit ausgeprägter Thetaund intermittierender rhythmischer Deltaaktivität (IRDA) sowie triphasische Wellen bis hin zum burstsuppression-Muster [26, 34]. Die Ausprägung dieser Veränderungen steht in Beziehung zur Beeinträchtigung des mentalen Status. Betrachtet man die EEG-

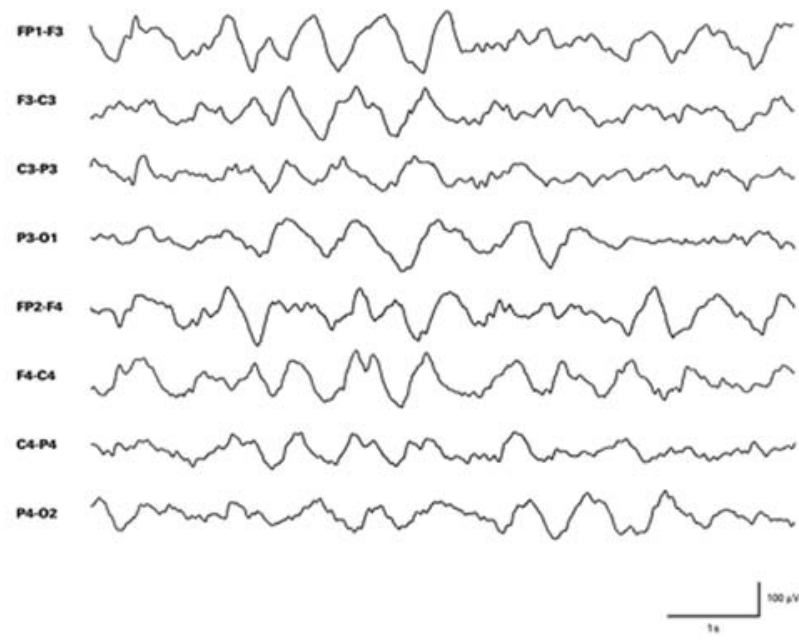

Abb. $\mathbf{3 a - c}$ Graduelle EEG-Veränderungen bei Multiorganversagen mit begleitender Enzephalopathie. In a) findet sich ein Delta-EEG mit schwerer Allgemeinveränderung, in b) periodische triphasische Wellen und in $\mathbf{c}$ ) ein Burst-Suppression-Muster. (Freundlicherweise von PD. Dr. Noachtar, Neurologische Klinik des Klinikum Großhadern, überlassen)

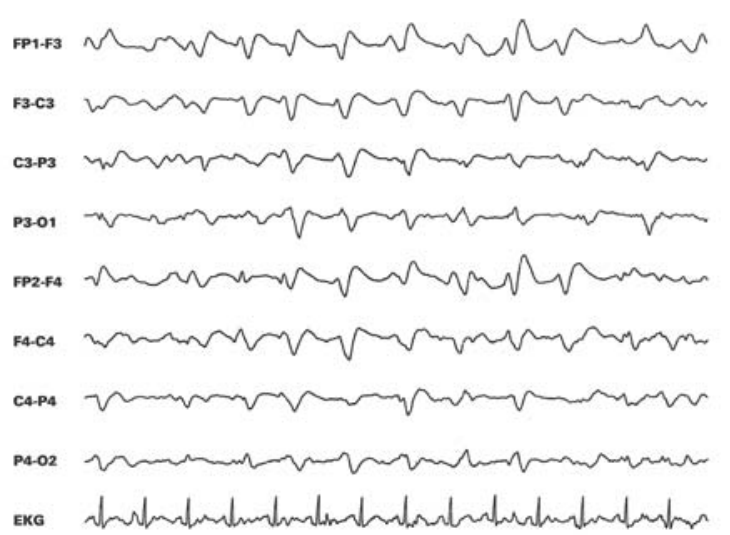

$\operatorname{lom}^{\operatorname{mox}}$

Abb. 3 b

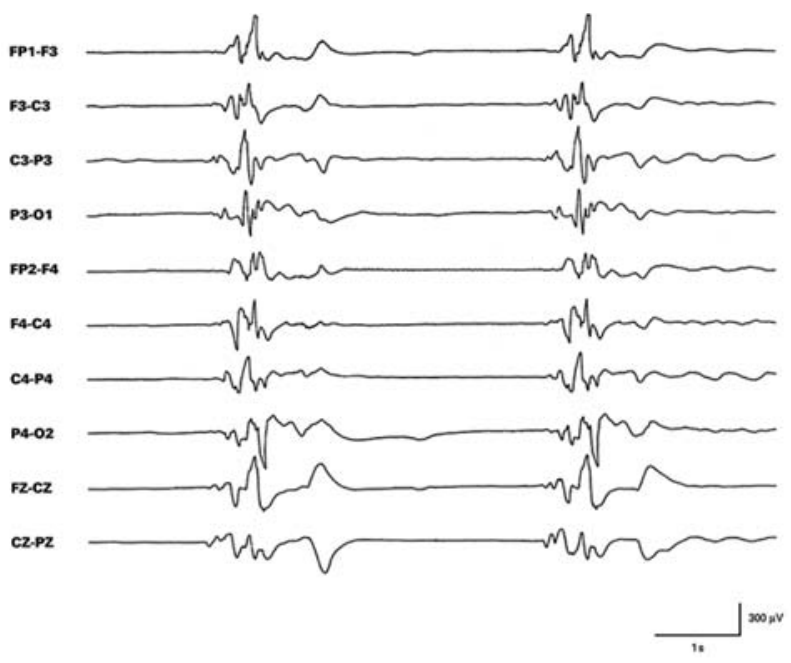

Abb. 3 c

Veränderungen in Verbindung mit dem klinischen Krankheitsbild, so lässt sich eine direkte Korrelation zur Mortalität feststellen [43].

Labor: Es sind keine spezifischen Befunde zu erheben. Nur in weniger als 50\% werden Erreger-positive Blutkulturen gefunden [27]. Im Bereich des ASStoffwechsels kommt es zu einer Störung, die eine Erhöhung der aromatischen und schwefelhaltigen und eine Verminderung der verzweigtkettigen AS zur Folge hat $[19,28,36]$.

Liquor: Die Liquorparameter sind meist unspezifisch verändert. Man findet normale oder allenfalls leicht erhöhte Eiweißwerte [4]. Wie auch im Blut zeigt sich eine erhöhte Konzentration an aromatischen bei erniedrigten Spiegeln an verzweigtkettigen Aminosäuren. In Korrelation zur Schwere der neurologischen Symptome werden erniedrigte AskorbatSpiegel gemessen [39]. 
SEP: Mit den somatosensibel evozierten Potentialen lässt sich der Verdacht einer septischen Enzephalopathie durch den Nachweis einer früh im Verlauf auftretenden kortikalen Desinhibition mit in bis $\mathrm{zu}$ $67 \%$ auftretender erhöhter kortikaler Reizantwort erhärten. In Kombination mit einer erniedrigten Reizantwort über Erb tritt diese fast ausschließlich in Folge einer schweren Sepsis mit Multiorganversagen in Erscheinung [34]. Es manifestiert sich eine Verlängerung kortikaler und subkortikaler Latenzen [45], einhergehend mit pathologisch konfigurierten meist verplumpten oder doppelgipfligen kortikalen Primärkomplexen sowie abgeflachten Amplituden. Die Mortalität steigt mit dem Ausmaß der Veränderungen [32].

CCT/MRT: Die Bildgebung hilft in der Diagnosefindung nicht weiter. In der Regel zeigen die Bilder einen Normalbefund. Nur in Einzelfällen werden unspezifische Veränderungen wie ein reversibles generalisiertes Marklagerödem oder hypodense Läsionen im Sinne von ischämischen Infarkten beobachtet $[2,35]$.

\section{Differentialdiagnosen}

Klinisches Bild und diagnostische Befunde der septischen Enzephalopathie sind nicht spezifisch für dieses Krankheitsbild. Dies kann zu Schwierigkeiten bei der Abgrenzung differentialdiagnostisch in Frage kommender Krankheitsbilder führen. Hierbei ist vor allem an sekundäre Enzephalopathien entzündlicher, vaskulärer, metabolischer, toxischer und infektiöser Genese zu denken. Besonders die Grenzen zu metabolischen Enzephalopathien sind dabei fließend. Diese sind in bis zu 29\% der Fälle Ursache neurologischer Komplikationen bei kritisch Kranken [1]. Bleck et al. zeigte in einer Untersuchung, dass bis $\mathrm{zu} 39 \%$ der metabolischen Enzephalopathien Folge einer Sepsis sind. Aber auch angeborene Stoffwechseldefekte, traumatische Schäden und psychiatrische Erkrankungen können sich ähnlich manifestieren $[8,35]$.

\section{Therapie}

Die Therapie der septischen Enzephalopathie entspricht der Therapie der Sepsis und des Multiorganversagens. Neben einer adäquaten Antibiotikatherapie gilt es eine Hypoxie, Hypokapnie und Ischämie $\mathrm{zu}$ vermeiden $[5,8,41]$. Dabei ist zu beachten, dass die Antibiotikatherapie eine vermehrte Zytokinausschüttung bewirken und so die septische Enzephalopathie begünstigen kann [13, 25]. Bei unzureichender selbständiger Atmung muss frühzeitig beatmet und ein adäquater Perfusionsdruck durch aggressive Therapie einer Hypotension, Volumenersatz und ge- gebenenfalls inotrope Substanzen aufrechterhalten werden [4]. Es wird kontrovers diskutiert, ob eine Ernährung mit erhöhtem Gehalt an verzweigtkettigen Aminosäuren, die dem Aminosäureungleichgewicht und der damit verbundenen gestörten Transmitterbildung entgegenwirken soll, sinnvoll ist. Im Gegensatz zur positiven Wirkung bei der hepatischen Enzephalopathie, konnte in einer Untersuchung von Meyenfeldt et al. keine Verbesserung des Zustandes von Patienten mit septischer Enzephalopathie unter Gabe von verzweigtkettigen AS erzielt werden $[36,40]$.

\section{Prognose}

Die septische Enzephalopathie geht mit einer erhöhten Mortalität einher. Dabei korreliert die Mortalität mit der Schwere der Enzephalopathie. Bei schweren Formen kann die Mortalität bis zu 50\% betragen, bei Patienten ohne Enzephalopathie 0-26\% $[37,44]$. Es zeigt sich allerdings ein erhebliches Erholungspotential des Gehirns, so dass die septische Enzephalopathie bei Überlebenden meist reversibel und eine vollständige Rückbildung möglich ist. Aber auch bleibende Hirnleistungsstörungen wurden beobachtet. Das EEG gilt als guter Prädiktor für das Überleben. Burst-suppression-Muster und fehlende Abwehrbewegungen auf Schmerzreize gehen mit einer schlechten Prognose einher [4].

\section{Enzephalopathie bei Multiorganversagen}

\section{Definition}

Es handelt sich um eine sich akut bis subakut entwickelnde diffuse Hirnschädigung durch Ausfall mehrerer systemischer Organe. In der Regel sind Niere, Leber, Lunge, Pankreas und das endokrine System in wechselnder Kombination betroffen [24]. Eine klare Zuordnung $\mathrm{zu}$ einer bestimmten gestörten Organfunktion ist in der Praxis ebenso schwierig wie die Abgrenzung von einer septischen Enzephalopathie bei zusätzlicher septischer Erkrankung. Die intensivmedizinische Realität ist die Entwicklung einer Enzephalopathie beim intubierten, beatmeten Patienten mit Ausfall der Nieren, schwerer Leberfunktionstörung und multiplen septischen Perioden, die zusätzlich durch Sedierung und Analgesie überdeckt wird.

\section{Symptomatik}

Die klinische Symptomatik unterscheidet sich nicht wesentlich von der der septischen Enzephalopathie, 
Tab. 2 Globale Zeichen der metabolischen Enzephalopathie

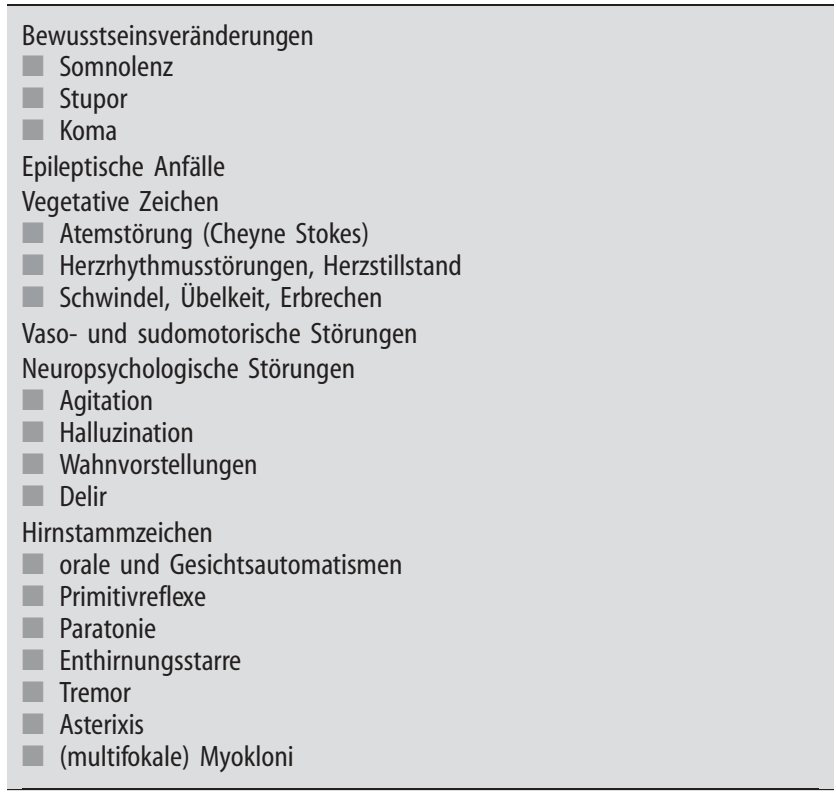

Nach Kunze 2002 (J Neurol 249:1150-1159)

\section{Tab. 3 Fokale Zeichen der metabolischen Enzephalopathie}

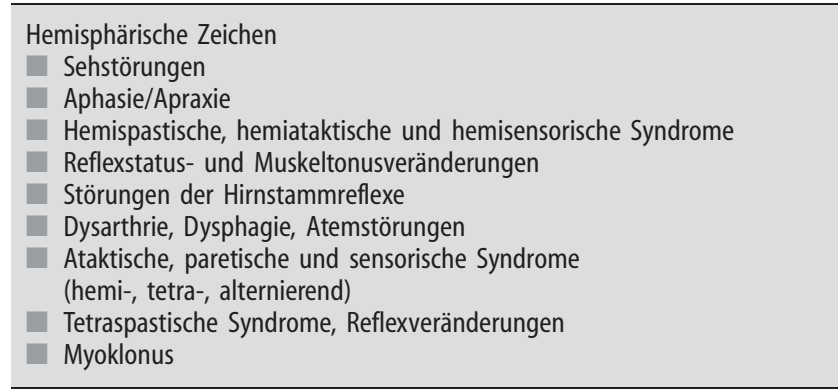

Nach Kunze 2002 (J Neurol 249:1150-1159)

d.h. im Vordergrund stehen die Entwicklung delirater Bilder und dann von Bewusstseinsstörungen als Ausdruck der globalen Hirnschädigung. Häufig findet sich ein schleichender Beginn mit Prodromi wie Antriebs- und Aufmerksamkeitsstörungen (vermehrte Ablenkbarkeit, Schläfrigkeit), Denkstörungen, Verkennungen (illusionäre Verkennungen), Desorientierung (v.a. räumlich und zeitlich) und Verwirrtheit [24]. Tabelle 2 fasst die wesentlichen Zeichen einer globalen Schädigung zusammen.

Allerdings können auch fokale klinische Zeichen auftreten, diese stehen meist im Hintergrund und werden von schwereren globalen Zeichen überlagert. Tabelle 3 fasst die fokalen Zeichen einer Enzephalopathie nach Multiorganversagen zusammen.

Die neurologische Untersuchung ergibt meist bilaterale Störungen der Motorik und Koordination, ei- ne allgemeine Hyperreflexie und als Besonderheiten Tremor (generalisiert, fein bis grobschlägig), Myoklonien (bilateral symmetrisch), bilaterale Asterixis (v.a. bei Leberversagen, das berühmte „Flügelschlagen"), Primitivreflexe, wie Schnauz- und Greifreflexe und generalisierte Veränderungen des Muskeltonus, während die Pupillomotorik lange erhalten bleibt.

\section{Pathophysiologie}

Ebenso wie bei der septischen Enzephalopathie gibt es eine Vielzahl von Mediatoren und nutritiven Aspekten, die mit der Pathophysiologie in Zusammenhang gebracht werden. Morphologische Veränderungen

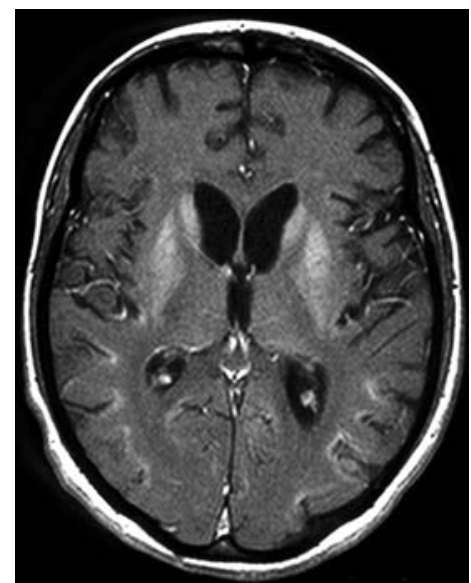

Abb. 4a-b Kernspintomographische Untersuchungen einer Patientin mit Zustand nach globaler Ischämie/Hypoxie. In a) sind Signalintensitätssteigerungen in den Basalganglien beidseits deutlich sichtbar und in b) diffuse laminäre, kortikale Nekrosen (jeweils T1 ohne KM). Beide Befunde sind typisch für globale Hypoxien (Z.n. Reanimation, schwerem Schock oder langdauernder Hypotonie/Hypoxie). (Freundlicherweise von Prof. Dr. Brückmann, Neuroradiologische Abteilung des Klinikum Großhadern, überlassen)

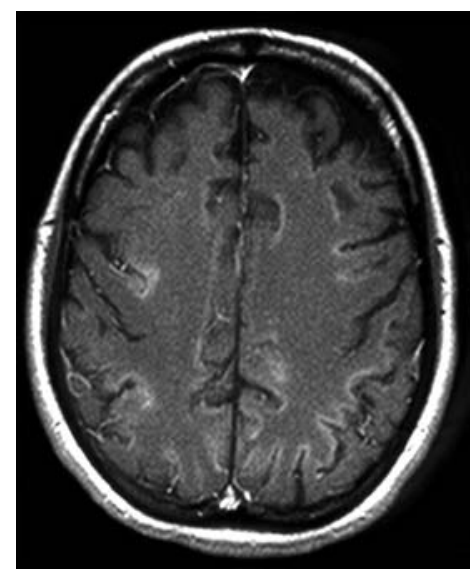

Abb. 4b 
Tab. 4 Stadien der hepatischen Enzephalopathie

\begin{tabular}{|c|c|c|c|c|}
\hline Grad & $\begin{array}{l}\text { Quantitatives } \\
\text { Bewusstsein }\end{array}$ & $\begin{array}{l}\text { Qualitatives } \\
\text { Bewusstsein }\end{array}$ & Neurologie & EEG \\
\hline 0 & Normal & Normal & Normal & Normal \\
\hline I & $\begin{array}{l}\text { Unruhe, } \\
\text { Schlafstörung }\end{array}$ & $\begin{array}{l}\text { Vergesslich, } \\
\text { verwirrt }\end{array}$ & $\begin{array}{l}\text { Tremor, } \\
\text { Dysdiadochokinese }\end{array}$ & Triphasisch \\
\hline \| & Lethargie & Orientierung $\downarrow$ & Asterixis & Triphasisch \\
\hline III & Somnolenz & Deutl. Orient. $\downarrow$ & Hyperreflexie & Triphasich \\
\hline IV & Koma & - & Babinski positiv & $\begin{array}{l}\text { Delta- } \\
\text { Rhythmus }\end{array}$ \\
\hline
\end{tabular}

Nach Ferenci et al. 2002 (Hepatology 35:716-721)

sind häufig blande und treten erst in Spätstadien auf. Begleitende globale ischämische Veränderungen sind beschrieben und können das klassische Bild mit symmetrischen bilateralen Basalgangliendefekten und sog. kortikalen laminären Nekrosen verursachen.

Spezielle pathophysiologische Aspekte werden bei den einzelnen Organsystemen beschrieben.

\section{Hepatische Enzephalopathie}

Die hepatische Enzephalopathie kann in Stadien von 0 bis 4 eingeteilt werden [9].

Neben Bewusstseinsstörungen als Leitsymptom kommt es in bis $\mathrm{zu} 30 \%$ der Fälle zu epileptischen Anfällen und in bis zu $80 \%$ zu einem Hirnödem. Die intrakranielle Drucksteigerung ist ein wesentlicher Grund für die hohe Mortalität der Patienten mit hepatischer Enzephalopathie (meist bei akuten Störungen, wie dem fulminanten Leberversagen) [9]. Pathophysiologisch ursächlich sind die Überschwemmung des Gehirns mit neurotoxischen Substanzen, wie Ammoniak und seinen Verbindungen, kurzkettige Fettsäuren, Mercaptane und Phenolen, die bei Leberversagen entstehen. Eine vermehrte Ammoniak-Diffusion ins Gehirn kann bei begleitender Hypokaliämie oder einer systemischen Alkalose beobachtet werden. Eine reduzierte hepatische Toxinelimination wird v.a. nach gastrointestinalen Blutungen oder akut nach portokavalem Shunt beobachtet. Eine zusätzliche Bedeutung hat die Aktivierung zentraler GABA-Rezeptoren (Dysbalance zwischen exzitatorischen und inhibitorischen Systemen) bei Patienten mit hepatischen Störungen [22].

In vielen Fällen mit akuter und sehr schwerer hepatischer Enzephalopathie besteht die einzig wirklich sinnvolle Behandlung in der Lebertransplantation [14].

\section{Renale Enzephalopathie}

Die urämische Enzephalopathie ist meist eine chronische Erkrankung, die aber bei Intensivaufenthalten oder akuten renalen Verschlechterungen dramatisch exazerbieren kann. Klassische Symptome sind Hyperventilation, Myoklonien und sich anschließende Bewusstseinsstörungen. Zusätzlich werden Hyperreflexie, Tremor und Hirnstammzeichen häufiger beobachtet. Ursächlich werden neben der Akkumulation renaler Ausscheidungsprodukte (insbesondere Harnstoff, Kreatinin, Kalzium, Kalium und Wasserstoffionen) auch immunologische Veränderungen (reduzierte $\mathrm{Ab}$ wehrsituation) angeschuldigt [24]. Inwieweit die Kalzium- und Kaliumverschiebungen die entscheidenden Störungen sind kann derzeit nur vermutet werden. Die Behandlung ist in der Regel die Dialyse mit angestrebter Normalisierung der Retentionsparameter.

\section{Elektrolytveränderungen}

Störungen des Wasser- und Elektrolythaushaltes sind häufig bei Intensivpatienten zu finden. Sie können zum einen Ausdruck eines neurologischen Grundleidens sein (im Sinne einer komplexen, zentralen Regulationsstörung), Beispiele sind das SIADH (Syndrom der inadequaten ADH-Sekretion), das zerebrale Salzverlustsyndrom (CSWS = cerebral salt wasting syndrome) oder der zentrale Diabetes insipidus. Andererseits können sie bei nicht neurologischen Ursachen zu neurologischen Symptomen führen (wie Hyponatriämie mit Bewusstseinsstörung und zerebralen Anfällen aufgrund einer exzessiven Diuretikagabe) [33]. Die häufigste klinisch relevante Elektrolystörung ist die Hyponatriämie auf die im Weiteren eingegangen werden soll. Andere seltenere Elektrolytstörungen können klinisch relevant sein und es wird auf die speziellere Literatur verwiesen $[18,33]$. Typische Symptome der Hyponatriämie sind Verhaltensstörungen, Verwirrtheit, Kopfschmerzen, Übelkeit und generalisierte Anfälle. Bei schweren Formen kommt es zur progredienten Bewusstseinsstörung. Muskelzuckungen und -krämpfe können, z.T. als Frühzeichen, auftreten. Allerdings sind die meisten Hyponatriämien klinisch asymptomatisch, lediglich sich rasch und ausgeprägt entwickelnde Störungen mit Serumnatriumwerten unter $125 \mathrm{mmol} / \mathrm{l}$ werden symptomatisch. Die Symptome sind der zerebralen Wasserverschiebung von extra- nach intrazellulär, also dem zellulären Hirnödem, zuzuschreiben. Bei langsamer Entwicklung haben dabei die intrazerebralen Zellen die Chance über Senkung ihrer eigenen Osmolalität (Verschiebungen von intrazellulären Kationen nach extrazellulär) dem nach intrazellulär gerichteten Wassereinstrom vorzubeugen. 


\section{Zentrale pontine Myelinolyse (ZPM)}

Ursprünglich wurde die ZPM als einzelne, scharf begrenzte demyelinisierende Läsion der Bahnen des Pons beschrieben.

Später wurden dann auch extrapontine Läsionen (in bis $\mathrm{zu} 10 \%$ der Fälle nachweisbar) als osmotische Myelinolysen zu der ZPM hinzugerechnet. Wie der Begriff der osmotischen Myelinolyse nahelegt, kann heute v.a. das Auftreten rascher Elektrolytschwankungen für das Auftreten der ZPM verantwortlich gemacht werden. Die ZPM wird v.a. nach Ausgleich einer Hyponatriämie oder bei Vorliegen einer akuten Hypernatriämie beobachtet. Der rasche Ausgleich einer Hyponatriämie ist dabei wohl für die Entwicklung der ZPM gefährlicher als die Hyponatriämie selbst. Besonders gefährdet sind Patienten mit Hyponatriämien und anderen schweren Grundleiden wie Lebererkrankungen, Verbrennungen, Mangelernährung, chronischem Alkoholismus und andere [18].

Typisch für das Auftreten der ZPM ist ein klinisch biphasischer Verlauf mit initial schwerem enzephalopathischem Bild mit Verwirrtheit, Desorientiertheit und Bewusstseinsstörungen, welches sich unter Therapie mit nachfolgendem Serumnatriumanstieg bessert. Dann folgt ein z.T. tagelanges Intervall mit klinischer Besserungstendenz bis hin zur Beschwerdefreiheit, gefolgt von den Symptomen der ZPM selbst, wie Hirnnervenausfällen, progredienter Tetraspastik, Dysarthrie, Schluckstörungen und anderen Hirnstammzeichen. Das Vollbild der ZPM führt zu einem locked-in-Syndrom.

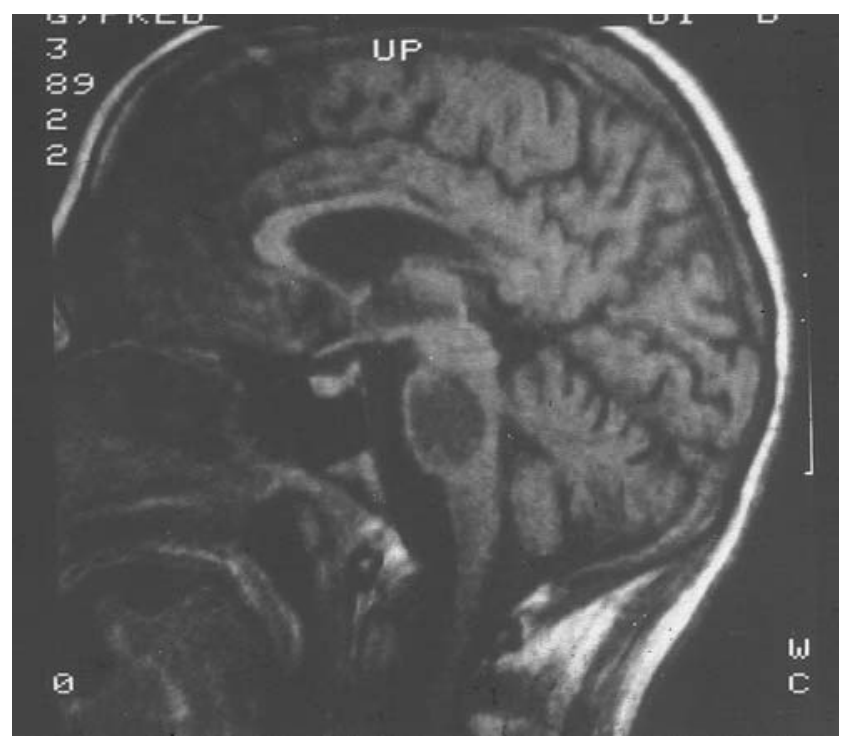

Abb. 5 T1-gewichtete Kernspintomographie eines 28-jährigen polytoxikomanen, drogenabhängigen Patienten mit schwerer Hyponatriämie (Serumnatrium $106 \mathrm{mmol} / \mathrm{l}$ ) und raschem Ausgleich auf $151 \mathrm{mmol} / \mathrm{l}$ bei begleitendem Leberversagen. (Freundlicherweise von Prof. Dr. Piepgras, Neuroradiologie des Universitätsklinikum Homburg, überlassen)

\section{SIADH (Syndrom der inappropriaten ADH-Sekretion)}

Klassisches Symptom ist neben der Hyponatriämie die erhöhte Natriumausscheidung im Urin (Natriumurinkonzentration über $20 \mathrm{mmol} / \mathrm{l}$ ), die Urinosmolalität übersteigt die Serumosmolalität wobei weder Volumenmangel noch -überschuss vorliegen. Ursachen sind neurologischerseits v. a. Subarachnoidalblutungen (SAB), Schädel-Hirn-Trauma (SHT), Meningoenzephalitiden, Tumoren des Hypothalamus oder ein Guillan-Barré-Syndrom. Prinzipiell kann es aber bei jeder raumfordernden zerebralen Erkrankung $\mathrm{zu}$ einem SIADH kommen. Neben Diuretika können viele verschiedene und sehr häufig rezeptierte Medikamente (z.B. Carbamazepin, Neuroleptika, Antidepressiva und nicht-steroidale Antiphlogistika) $\mathrm{zu}$ einem SIADH führen. Vor allem bei älteren $\mathrm{Pa}$ tienten findet sich ein SIADH sehr häufig und führt in ca. $10 \% \mathrm{zu}$ zerebralen Anfällen und in rund $40 \%$ der Fälle zu einer Bewusstseinstrübung.

Patienten mit SIADH sollten durch Wasserrestriktion und Gabe geringer Mengen von Kochsalz behandelt werden. Der Einsatz oraler Vasopressinantagonisten kann zur kausalen Behandlung des SIADH hilfreich sein.

\section{Behandlung der Hyponatriämie}

Asymptomatische Patienten werden mit Flüssigkeitsrestriktion (ca. $1500 \mathrm{ml}$ Zufuhr) und oraler Natriumgabe behandelt, symptomatische Patienten sollten etwas aggressiver behandelt werden, bis die Serumnatriumwerte ca. $125 \mathrm{mmol} / \mathrm{l}$ erreicht haben. Bei akuten Hyponatriämien sollten ein Serumnatriumanstieg von maximal $0,5 \mathrm{mmol} / \mathrm{l}$ pro $\mathrm{h}$ nicht überschritten werden (Gabe von 0,9\% NaCl-Lsg. i.v.) und bei ca. $125 \mathrm{mmol} / \mathrm{l}$ sollte eine Behandlungspause erfolgen [16]. Eine Normalisierung der Serumnatriumwerte sollte initial nicht angestrebt werden. Chronische Hyponatriämien sollten noch langsamer und vorsichtiger ausgeglichen werden (über die Gefahr einer ZPM s. o.). Trotzdem scheint es in Einzelfällen mit Einklemmungsgefahr bei schwerem Hirnödem oder Anfällen gerechtfertigt, schneller $(1,5 \mathrm{mmol} / \mathrm{h}$ Serumnatriumwert) auszugleichen. Über $20 \mathrm{mmol} / \mathrm{l}$ Serumnatriumänderung in $24 \mathrm{~h}$ sollten aber vermieden werden. Die Serumnatriumwerte müssen stündlich bestimmt werden. Wichtig scheint die alte Regel, dass eine sich akut entwickelnde Hyponatriämie auch akut ausgeglichen werden kann, während eine eher chronisch, sich schleichend entwickelnde Hyponatriämie nur langsam ausgeglichen werden sollte. 


\section{Critical illness Polyneuropathie}

\section{Definition}

Die critical illness Polyneuropathie (CIP) ist eine reversible akut auftretende vorwiegend axonale Polyneuropathie (PNP), die de novo während schwerer, intensivbehandlungspflichtiger Erkrankungen auftritt, insbesondere bei Sepsis und Multiorganversagen.

\section{Epidemiologie}

Ca. 35\% der Patienten mit Sepsis zeigen im Laufe ihrer Erkrankung Symptome einer CIP. Der tatsächliche Anteil der Patienten mit CIP liegt jedoch mit $70 \%$ weit höher wie elektrophysiologische Untersuchungen in einer prospektiven Studie zeigten [21]. Bei Patienten, die über 3 Wochen intensivtherapiert werden, findet man die CIP sogar in bis $\mathrm{zu} 90-100 \%$ der Fälle [34].

\section{Symptomatik}

Die CIP tritt mit einer Latenz von Tagen bis Wochen später auf als die septische Enzephalopathie. Häufigste Manifestation ist die verzögerte Entwöhnung vom Respirator. Hinzu kommen symmetrische distal betonte schlaffe Paresen mit abgeschwächten oder fehlenden Reflexen, die in der Regel zuerst die Beine betreffen und bis hin zur Tetraplegie führen können [26]. Die Gesichtsmuskulatur bleibt meist ausgespart. Die CIP geht fakultativ mit diskreten Sensibilitätsstörungen einher [23].

Patienten, die von einer CIP betroffen sind, zeigen eine höhere Sekundärkomplikationsrate, was $\mathrm{zu}$ einem längeren Aufenthalt auf Intensivstation führt und eine verzögerte Mobilisation und neurologischer Rehabilitation nach sich zieht [26].

\section{Pathophysiologie}

Bis heute ist es nicht gelungen, die genaue Ursache und die Faktoren, die zur Entwicklung einer CIP beitragen, aufzuklären. Man geht von einer multifaktoriellen Genese aus, wobei Sepsis, Multiorganversagen, intensivpflichtige Versorgung mit langandauernder Beatmung und extrakorporaler Kreislauf prädisponierend sind $[4,21]$.

Nach Aktivierung proinflammatorischer Mediatoren wie Zytokine und Radikale lässt sich neben direkten myelin- oder axonschädigenden und myolytischen Eigenschaften eine Zunahme der Gefäßper- meabilität beobachten, die $\mathrm{zu}$ einem endoneuralen Ödem führt. Dies hat einen Verschluss von Kapillaren und damit eine Minderperfusion und Hypoxie zur Folge. Dabei kommt es zu einem Untergang von Axonen mit resultierender neurogener Muskelatrophie [31, 42].

Witt et al. fanden in einer prospektiven Studie mit 43 Patienten eine Korrelation zwischen erhöhter Blutglukose und erniedrigtem Serumalbumin und einer verminderten Funktion peripherer Nerven. Die Hyperglykämie führt über die Erhöhung des Gefäßwiderstandes $\mathrm{zu}$ einem herabgesetzten Blutfluss in den Vasa nervorum. Die dadurch induzierte endoneurale Hypoxie zieht eine Schädigung der Mitochondrien und des axonalen Transportes nach sich.

Die bei Sepsis freigesetzten histaminähnlichen Substanzen sind dafür verantwortlich, dass es zur Störung der BHS kommt, was durch eine Verlagerung von Albumin ins Interstitium zum Ausdruck kommt [42].

Diverse Studien belegen einen Zusammenhang zwischen dem Auftreten einer axonalen Neuropathie und der Behandlung von Patienten mit neuromuskulär blockierenden Substanzen $[15,30]$. Werden diese mit Steroiden kombiniert eingesetzt, so verursachen sie eine Myopathie mit Verlust der dicken Myosinfilamente [6].

$\mathrm{Zu}$ den weiteren möglichen Ursachen, die zur Entwicklung einer CIP führen können, zählen der therapeutische Einsatz von Aminoglykosiden, metabolische Störungen [30], Hypoxie und Blutdruckabfall.

\section{Diagnostik}

Labor und Liquor: Die Labor- und Liquordiagnostik hilft bei der Diagnosefindung nicht weiter. Man findet allenfalls unspezifisch veränderte Werte wie eine leicht erhöhte Zellzahl und ein leicht erhöhtes Eiweiß im Liquor. Die Werte für die Kreatinkinase liegen in der Regel im Normbereich [21]. Allerdings wurden auch mehrfach Werte um $200 \mathrm{U} / \mathrm{l}$ gemessen [23].

Elektrophysiologie: Die Elektrophysiologie ist die hilfreichste Methode, um periphere Nervenläsionen $\mathrm{zu}$ diagnostizieren. Gerade bei intensivpflichtigen Patienten, die klinisch oft nur eingeschränkt beurteilbar sind, spielt sie eine entscheidende Rolle.

Die CIP, die mit einer axonalen Degeneration vorwiegend motorischer Nervenfasern einhergeht, zeigt typischer Weise in den ersten Wochen nach Symptombeginn normale oder nahezu normale Nervenleitgeschwindigkeiten und distal-motorische Latenzen bei gleichzeitig verbreiterten, amplitudengeminderten Summenaktionspotentialen [21]. Nur vereinzelt findet man verlängerte F-Wellenlatenzen, die auf eine proximale Demyelinisierung hinweisen [42]. 
Erst nach ca. 14 Tagen tritt in der Elektromyographie eine pathologische Spontanaktivität im Sinne von Fibrillationspotentialen und positiven scharfen Wellen auf als Zeichen für eine vor allem proximal betonte Denervierung [26].

Somatosensibel evozierte Potenziale (SEP): Mit den SEP sind gleichzeitig Aussagen über periphere und zentrale Reizleitungsstörungen möglich. Sie sind damit sehr wertvoll zur Überwachung auf Intensivstation. Bei Ableitung über dem Erb'schen Punkt zeigen sich erniedrigte Reizantworten bei nur leichter peripherer Leitungsverzögerung [34].

Muskelbiopsie: Durch die axonale Degeneration der Nerven kommt es zu einer neurogenen Muskelatrophie, häufig kombiniert mit sekundär myopathischen Veränderungen im Sinne einer nekrotisierenden Myopathie wie bei der Critical illness Myopathie [34].

\section{Differentialdiagnose}

Bevor bei Paresen und Entwöhnungsschwierigkeiten vom Respirator eine CIP diagnostiziert werden kann, müssen diverse, sich ähnlich manifestierende Erkrankungen ausgeschlossen werden. Neben der nachfolgend ausführlich beschriebenen Critical illness Myopathie sind in erster Linie Neuropathien wie das Guillain-Barré-Syndrom, die spinale Muskelatrophie, Motoneuronerkrankungen infektiöser oder vaskulärer Natur, paraneoplastische und toxische Polyneuropathien sowie neuromuskuläre Erkrankungen wie die Myasthenia gravis, das Lambert-Eaton-Syndrom, medikamentös oder toxisch bedingte neuromuskuläre Übertragungsstörungen und Myopathien wie die Steroidmyopathie, die maligne Hyperthermie und Myositiden zu nennen [26].

\section{Therapie}

Wie bei der septischen Enzephalopathie gibt es auch für die CIP keine spezifische Therapie. Als mögliche Therapie bleibt die Behandlung der ätiologisch ursächlichen Sepsis und des Multiorganversagens zusammen mit einer frühzeitig begonnenen und konsequent durchgeführten krankengymnastischen Übungsbehandlung. Der Einsatz von hochdosierten Immunglobulinen ist umstritten. In einer Pilotstudie an drei Patienten von Wijdicks et al. haben Immunglobuline keinen positiven Effekt. Mohr et al. kamen anhand klinischer Beobachtungsstudien $\mathrm{zu}$ dem Schluss, dass die frühzeitige Gabe von Immunglobulinen die Entwicklung einer CIP verhindern kann bzw. die Ausprägung der Symptome zu lindern vermag. Von 8 Patienten mit Sepsis, die mit Immunglobulinen über drei Tage behandelt wurden, entwickelte keiner eine CIP, wohingegen von den 8 Patienten aus der Kontrollgruppe ohne Immunglobulinbehandlung 7 eine CIP entwickelten. Präventiv sollte die Körpertemperatur unter $40{ }^{\circ} \mathrm{C}$ gehalten sowie der Einsatz von nicht depolarisierenden Muskelrelaxanzien oder Aminoglykosiden in Kombination mit Steroiden vermieden werden, da diese einer Entwicklung einer CIP förderlich sein können [21, 26].

\section{Prognose}

Die Prognose von Patienten mit Sepsis und Multiorganversagen ist bei einer Mortalität zwischen 48-98\% in Abhängigkeit von der Anzahl der versagenden Organe nach wie vor schlecht. Inwieweit die CIP die Mortalität erhöht ist weiterhin unklar. Es hat sich jedoch gezeigt, dass Patienten mit einer CIP ein erhöhtes Risiko für Sekundärkomplikationen wie Pneumonie, tiefe Beinvenenthrombose und Lungenembolie haben, länger beatmet werden müssen und eine längere Rehabilitationsphase aufweisen [21]. In einer Untersuchung von Thiele et al. [38] starben nach einer Herz-Operation 4 von 7 Patienten mit und nur 3 von 37 ohne CIP.

Der Verlauf der CIP ist monophasisch und nach Ausheilung der ätiologisch ursächlichen Sepsis und des Multiorganversagens selbstlimitierend. Je nach Schwere der Polyneuropathie kann eine Restitution Wochen bis Monate dauern. In ca. 50\% der Fälle kommt es zur vollständigen Rückbildung der Symptome, bei schwerer CIP bleibt die Rückbildung aber häufig unvollständig [42].

\section{Critical illness Myopathie}

\section{Definition}

Von einer Critical Illness Myopathie spricht man, wenn unspezifische myopathische Veränderungen das histopathologische Bild kritisch kranker Patienten mit Muskelschwäche dominieren [21].

\section{Epidemiologie}

Bei $50-80 \%$ der kritisch Kranken tritt eine muskuläre Beteiligung auf [26]. In den auf Muskelbiopsien gestützten Untersuchungen wird die Inzidenz der CIM mit durchschnittlich 32\% angegeben [34].

\section{Symptomatik}

Wie die CIP tritt auch die CIM mit einer Latenz von Tagen bis Wochen auf. Klinisch zeigen die Patienten 
eine generalisierte Muskelschwäche bis hin zur Tetraplegie, die vor allem proximal betont ist und nur selten die Gesichtsmuskulatur mit einbezieht [26]. Bei teigig-weicher Muskulatur, die zwar spät, aber häufig atrophiert, sind die Muskeleigenreflexe abgeschwächt oder ganz ausgefallen [34]. Oft bedeutet die CIM, v. a. auch in Kombination mit einer CIP, für die Patienten eine verzögerte Entwöhnung vom Respirator, längere Intensivaufenthalte, erhöhte Komplikationsraten und eine verzögerte neurologische Rehabilitation [21].

\section{Pathophysiologie}

Welche Pathophysiologie der CIM mit ihren unterschiedlichen histologischen Manifestationsformen (s. dazu Biopsie) zugrunde liegt ist weiterhin unklar. Unbestritten ist allerdings, dass der Einsatz einer Kombination von Steroiden mit nicht-depolarisierenden Muskelrelaxanzien bei beatmeten Patienten mit einem hohen Risiko einhergeht, eine CIM zu entwickeln [34]. Man vermutet unter anderem eine Faserschädigung durch die bei Sepsis und Multiorganversagen auftretende Minderperfusion und Hypoxie. Neben den bereits erwähnten Muskelrelaxanzien und Kortison haben auch Aminoglykoside und Inaktivität eine verstärkende Wirkung auf die Schädigung [26]. In einer Studie von De Letter et al. konnte gezeigt werden, dass eine geringe Anzahl aktivierter Leukozyten, die sowohl proinflammatorische (TNF alpha, IL-1) als auch antiinflammatorische Zytokine (IL-10) produzieren, den Skelettmuskel infiltrieren. Dabei wird vermutet, dass die Balance der Leukozytenaktivitäten eine große Rolle bei der Entwicklung einer Myopathie spielt [7].

\section{Diagnostik}

Labor und Liquor: Es finden sich keine für die CIM spezifisch veränderten Parameter. Die CK-Werte können je nach Erscheinungsform der CIM erhöht sein, bewegen sich aber meist im Normbereich [34].

EMG: Die durch die Sepsis und Multiorganversagen verursachte Myopathie manifestiert sich im EMG durch myopathische Muster. Das Auftreten einer Muskelnekrose kann eine abnorme Spontanaktivität des Muskels verursachen, welche nicht immer klar von der durch Denervation hervorgerufenen Spontanaktivität $\mathrm{zu}$ unterscheiden ist. Dabei sprechen sehr früh auftretende Spontanaktivitäten eher für die CIM. Auch polyphasische und niedrigamplitudige Muskelpotentiale treten sowohl bei früher Reinnervation als auch bei primären Muskelerkrankungen auf $[4,34]$.
NLG: Die direkte faradische Stimulation des Muskels, die mit der Membranerregbarkeit der Muskelfasern korreliert, soll hilfreich sein, die CIM von der CIP abzugrenzen. Nur bei der CIM zeigt sich diese schon nach wenigen Tagen reduziert [34].

Biopsie: Muskelbiopsien können bei differentialdiagnostischen Schwierigkeiten indiziert sein. Bei Intensivpatienten können dabei drei Hauptformen der Myopathie unterschieden werden [34].

Kachektische Myopathie

- Sie zeichnet sich durch unterschiedliche Muskelfasergrößen, Einzelfaseratrophien und -nekrosen (v.a. Typ-II-Fasernekrosen), fettige Degeneration und Fibrose aus.

- Sie tritt infolge von Inaktivität, Katabolie und Kachexie auf.

Akute Myopathie der dicken Filamente

- Es zeigt sich ein selektiver Verlust von Myosinfilamenten.

- Sie kommt vor allem bei Patienten unter hochdosierter Glukokortikoidtherapie und neuromuskulärer Blockade vor.

Nekrotisierende Myopathie

- In deren Rahmen manifestieren sich panfaszikuläre Muskelfasernekrosen mit Zeichen der Vakuolisierung und Phagozytose.

- Man findet sie bei schwersten intensivmedizinischen Krankheitsbildern.

\section{Differentialdiagnose}

Die Differentialdiagnosen der CIM entsprechen denen der CIP. Bevor also die Diagnose der CIM gestellt werden kann, muss die CIP sowie diverse Neuropathien, neuromuskuläre Erkrankungen und Myopathien ausgeschlossen werden (siehe Differentialdiagnosen der CIP). Zur Diagnosefindung kann hilfreich sein, dass sich nur bei der CIM eine verlangsamte Muskelfaserleitung finden lässt, basierend auf Störungen von Natrium- und Chlorid-Kanälen [26].

\section{Therapie}

Die Therapie der CIM sollte eine Kombination aus Ursacheneliminierung und Symptombehandlung sein. Zum einen gilt es, die Sepsis und das Multiorganversagen zu therapieren, eine kombinierte Anwendung von nicht-depolarisierenden Muskelrelaxanzien und Steroiden bei beatmeten Patienten zu vermeiden und zum anderen die Muskelschwäche krankengymnastisch zu behandeln. Eine sich dem Klinikaufenthalt unmittelbar anschließende Rehabilitation trägt dazu bei, das Outcome der Patienten weiter zu verbessern [34]. 


\section{Prognose}

Die Prognose der CIM ist abhängig von der Schwere der Myopathie. Leichte Formen zeigen eine spontane und schnelle Rückbildung der Symptome nach Ausheilung der ätiologisch ursächlichen Sepsis und des Multiorganversagens. In schweren Fällen ist die Prognose eher schlecht, auch aufgrund des erhöhten Risikos für Sekundärkomplikationen [3].

Generell kann man sagen, dass das Outcome von Patienten mit CIM schlechter ist als das von Patienten ohne CIM [34].

\section{Vegetative Störungen}

\section{Definition}

Viele Intensivpatienten leiden unter begleitenden Störungen des autonomen Nervensystems, hierbei kommt es meist durch endogene Katecholaminproduktion oder externe Stimulierung und Ersatztherapie zu einer starken sympathikotonen Erregung. Die Symptome sind vielfältig und wie bei Fieber oder Störungen des Tag-Nacht-Rhythmus z.T. nur verzögert als Störungen des vegetativen Nervensystems von z.B. Infektionen zu unterscheiden.

\section{Epidemiologie}

Es liegen keine genauen Angaben $\mathrm{zu}$ vegetativen Störungen bei Intensivpatienten vor. Man kann davon ausgehen, dass bei den meisten Intensivpatienten mit längerem Intensivaufenthalt begleitende Störungen des vegetativen Nervensystem vorliegen.

\section{Symptomatik}

Diese ist vielfältig und umfasst:

Kardiale Symptome wie:

- Einschränkung der Herzfrequenzvariabilität sowie der Baro- und Chemoreflexsensitivität

- EKG-Veränderungen: long-QT, cerebral T-Waves, Kammerarrhythmien, torsade de points

- kardiovaskuläre Symptome wie Hypertonie, Brady- und Tachykardien, auch im Wechsel

- Herzinfarkt oder neurogenic stunned myocardium

Neurogenes Lungenödem

Metabolische Störungen (Hypermetabolismus,

Katabolismus)

Elektrolytstörungen (Cerebral Salt Wasting

Syndrome, SIADH)
Fieber

Stress mit Unruhe und Ängstlichkeit

Störungen des Tag-Nacht-Rhythmus

Wie erwähnt kann es im Einzelfall schwierig sein, die vegetativen Symptome von anderen Auslösern zu trennen.

\section{Therapie}

Die Therapie des Grundleidens ist wie bei den meisten neurologischen Komplikationen der Intensivtherapie am wichtigsten. In Einzelfällen kann eine niedrig dosierte Betablockertherapie zu einer Hemmung des überschießenden Sympathikotonus und damit zu einer Verbesserung führen [17].

\section{Vorschläge für diagnostisches und therapeutisches Vorgehen}

Allgemeine diagnostische und therapeutische Maßnahmen sind schwer zu treffen, bei den obigen Punkten wurde auf die Diagnostik und Therapien im Speziellen hingewiesen.

Die zentralen, peripheren und vegetativen neurologischen Komplikationen kritischer kranker Patienten bei Sepsis und Multiorganversagen können durch viele unspezifischen Symptome in Erscheinung treten und erst nach Ausschluss sich ähnlich manifestierende Erkrankungen diagnostiziert werden. Dabei werden sie häufig aufgrund anderer, im Vordergrund stehender schwerwiegender Erkrankungen sowie Sedierung und Analgesie überdeckt. Deshalb sollte bei längeren Aufenthalten auf einer Intensivstation immer auch an die Möglichkeit des Auftretens der beschriebenen neurologischen Komplikationen gedacht werden.

Häufig helfen die in der neurologischen Intensivmedizin routinemäßig durchgeführten Untersuchungen wie Labor- und Liquoruntersuchungen sowie bildgebende Verfahren bei der Diagnosefindung nicht weiter. Bei Verdacht sollte deshalb, begleitend zum klinischen Erscheinungsbild, die Diagnose mittels EEG und elektrophysiologischer Zusatzdiagnostik gesichert werden. Wichtig ist dabei die gute $\mathrm{Zu}$ sammenarbeit zwischen Intensivmedizinern und den neurologischen Fachkollegen. Das Hinzuziehen von Neurologen mit spezieller Erfahrung ist in der neurologischen Intensivmedizin hilfreich, um schwierige Einzelpunkte zu klären.

Die frühzeitige und adäquate Therapie des Grundleidens stellt die wichtigste Voraussetzung für eine Rückbildung der neurologischen Symptome dar. 
Bei florider neuromuskulärer Erkrankung sollte vorerst von Entwöhnungsversuchen vom Respirator und Mobilisationsversuchen abgesehen werden. Eine zusätzliche medikamentöse Beeinflussung der neuromuskulären Übertragung durch v.a. nicht-depolarisierende Muskelrelaxanzien in Kombination mit Steroiden sollte, wenn möglich, vermieden werden.
Bei Besserung der Akutsymptomatik kann mit einer krankengymnastischen Übungsbehandlung begonnen werden, die, konsequent durchgeführt, wesentlich zu einem guten Outcome der Patienten beiträgt.

\section{Literatur}

1. Bleck TP, Smith MC, Pierre-Louis SJC, Jares JJ, Murray J, Hansen CA (1993) Neurologic complications of critical medical illness. Crit Care Med 21:98-103

2. Bogdanski R. Blobner M, Hanel F, Kochs E (1999) Septic encephalopathy. Anasthesiol Intensivmed Notfallmed Schmerzther 34:123-130

3. Bolton CF (1996) Sepsis and the systemic inflammatory response syndrome: neuromuscular manifestations. Crit Care Med 24:1408-1416

4. Bolton CF, Young GB, Zochodne DW (1993) The neurological complications of sepsis. Ann Neurol 33:94-100

5. Bowton DL, Bertels NH, Prough DS, Stump DA (1989) Cerebral blood flow is reduced in patients with sepsis syndrome 1. Crit Care Med 17:399403

6. Danon MJ, Carpenter S (1991) Myopathy with thick filament (myosin) loss following prolonged paralysis with vecuronium during steroid treatment. Muscle Nerve 14:1131-1139

7. De Letter MA, van Doorn PA, Savelkoul HF, Laman JD, Schmitz PI, Op de Coul AA, Visser LH, Kros JM, Teepen JL, van der Meche FG (2000) Critical illness polyneuropathy and myopathy (CIPNM): evidence for local immune activation by cytokineexpression in the muscle tissue 1. J Neuroimmunol 106:206-213

8. Eggers V, Schilling A, Kox WJ, Spies C (2003) Septic encephalopathy. Diagnosis und therapy. Anaesthesist 52: 294-303

9. Ferenci P, Lockwood A, Mullen K, Tarter R, Weissenborn K, Blei AT (1998) Hepatic encephalopathy-definition, nomenclature, diagnosis, and quantification: final report of the working party at the 11th World Congresses of Gastroenterology. Vienna, Hepatology 1(35):716-721

10. Fischer JE, Baldessarini RJ (1971) False neurotransmitters and hepatic failure. Lancet 6(2):75-80
11. Freund HR, Muggia-Sullam M, LaFrance R, Holroyde J, Fischer JE (1986) Regional brain amino acid and neurotransmitter derangements during abdominal sepsis and septic encephalopathy in the rat. The effect of amino acid infusions. Arch Surg 3(121):209-216

12. Freund HR, Muggia-Sullam M, Peiser J, Melamed E (1985) Brain neurotransmitter profile is deranged during sepsis and septic encephalopathy in the rat. J Surg Res 1(38):267-271

13. Fügener $\mathrm{K}$, Rommelspacher $\mathrm{H}$, Kox WJ, Speis CD (2001) The role of TNF alpha and norharman in septic encephalopathy during ventilator-associated pneumonia treated with antibiotics. Crit Care Med [Suppl]

14. Gill RQ, Sterling RK (2001) Acute liver failure. J Clin Gastroenterol 2(33):191-198

15. Gooch JL, Suchyta MR, Balbierz JM, Petajan JH, Clemmer TP (1991) Prolonged paralysis after treatment with neuromuscular junction blocking agents. Crit Care Med 19:1125-1131

16. Gross P, Reimann D, Neidel J, Doke C, Prospert F, Decaux G, Verbalis J, Schrier RW (1998) The treatment of severe hyponatremia. Kidney Int 2(Suppl 64):6-11

17. Hamann G, Haass A, Schimrigk $K$ (1993) Beta-blockade in acute aneurysmal subarachnoid haemorrhage. Acta Neurochir 2(121):119-122

18. Hamann GF (2001) Bedeutungen von Störungen des Wasser- und Elektrolythaushaltes in der neurologischen Intensivmedizin. Aktuelle Neurologie 28:103-113

19. Hasselgren PO, Fischer JE (1986) Septic encephalopathy. Etiology and management. Intensive Care Med 1(12):13-16

20. Houtchens BA, Westenskow DR (1984) Oxygen consumption in septic shock: collective review. Circ Shock 13:361-384
21. Hund E (1999) Critical-illness-Polyneuropathie und -Myopathie In: Schwab S, Krieger D, Müllges W, Hamann G, Hacke W (eds) Neurologische Intensivmedizin. Springer, Berlin Heidelberg New York, pp 699-709

22. Jones EA (2000) Pathogenesis of hepatic encephalopathy. Clin Liver Dis 1(4):467-485

23. Kuhn Ch, Werdan K (2002) Therapie der Organdysfunktion von Gehirn, autonomem und peripherem Nervensystem sowie Skelettmuskulatur. In: Eckart, Forst, Burchardi (Eds) Intensivmedizin

24. Kunze K (2002) Metabolic encephalopathies. J Neurol 249:1150-1159

25. Lepper PM, Held TK, Schneider EM, Bolke E, Gerlach H, Trautmann M (2002) Clinical implications of antibiotic-induced endotoxin release in septic shock. Intensive Care Med, 2(28):824-833

26. Lindner A, Kappen K, Zierz S (1998) Acute encephalopathy, polyneuropathy and myopathy in the critically ill patient. Internist (Berl) 39:485-492

27. Meakins JL, Wicklund B, Forse RA, McLean AP (1980) The surgical intensive care unit: current concepts in infection. Surg Clin North Am 4(60): 117-132

28. Mizock BA, Sabelli HC, Dubin A, Javaid JI, Poulos A, Rackow EC (1990) Septic encephalopathy evidence for altered phenylalanine metabolism and comparison with hepatic encephalopathy. Arch Intern Med 150:443-449

29. Neugebauer E, Dimmeler S, Troidl H (1995) Mediator systems and infection. Chirurg 66:2-10

30. Op de Coul AA, Lambregts PC, Koeman J, van Puyenbroek MJ, Ter Laak HJ, Gabreels-Festen AA (1985) Neuromuscular complications in patients given Pavulon (pancuronium bromide) during artificial ventilation. Clin Neurol Neurosurg 87:17-22

31. Op de Coul AA, Verheul GA, Leyten AC, Schellens RL, Teepen JL (1991) Critical illness polyneuromyopathy after artificial respiration 5. Clin Neurol Neurosurg 5(93):27-33 
32. Pfadenhauer K, Rittner J, Stohr M (1997) Sepsis: impaired consciousness of unknown origin as the initial symptom. Clinical aspects and pathophysiology of septic encephalopathy. Comment on the contribution by $\mathrm{S}$ Schwarz (et al) Nervenarzt 68:10081009

33. Riggs JE (1989) Neurologic manifestations of fluid and electrolyte disturbances. Neurol Clin 2(7):509-523

34. Schegelmann K (2002) Neurologische Komplikationen der Sepsis. In: Eckart, Forst, Burchardi (eds) Intensivmedizin

35. Schwarz S, Schwab S, Fabian CW, Schellinger P, Orberk E, Hund E (1997) Infection: impaired consciousness as the initial symptom. Clinical and pathophysiologic aspects of septic encephalopathy. Nervenarzt 68:292-297

36. Sprung CL, Cerra FB, Freund HR, Schein RM, Konstantinides FN, Marcial EH, Pena M (1991) Amino acid alterations and encephalopathy in the sepsis syndrome. Crit Care Med 19: 753-757
37. Sprung CL, Peduzzi PN, Shatney $\mathrm{CH}$, Schein RM, Wilson MF, Sheagren JN, Hinshaw LB (1990) Impact of encephalopathy on mortality in the sepsis syndrome. The Veterans Administration Systemic Sepsis Cooperative Study Group. Crit Care Med 1(18):801-806

38. Thiele RI, Jakob H, Hund E, Tantzky S, Keller S, Kamler M, Herold U, Hagl S (2000) Sepsis and catecholamine support are the major risk factors for critical illness polyneuropathy after open heart surgery. Thorac Cardiovasc Surg 48:145-150

39. Voigt K, Kontush A, Stuerenburg HJ, Muench-Harrach D, Hansen HC, Kunze K (2002) Decreased plasma and cerebrospinal fluid ascorbate levels in patients with septic encephalopathy. Free Radic Res 36:735-739

40. von Meyenfeldt MF, Soeters PB, Vente JP, van Berlo CL, Rouflart MM, de Jong KP, van der Linden CJ, Gouma DJ (1990) Effect of branched chain amino acid enrichment of total parenteral nutrition on nitrogen sparing and clinical outcome of sepsis and trauma: a prospective randomized double blind trial. Br J Surg 1(77): 924-929
41. Wijdicks EF, Stevens M (1992) The role of hypotension in septic encephalopathy following surgical procedures. Arch Neurol 49:653-656

42. Witt NJ, Zochodne DW, Bolton CF, Grand'Maison F, Wells G, Young GB, Sibbald WJ (1991) Peripheral nerve function in sepsis and multiple organ failure. Chest 99:176-184

43. Young GB, Bolton CF, Archibald YM, Austin TW, Wells GA (1992) The electroencephalogram in sepsis-associated encephalopathy. J Clin Neurophysiol 9:145-152

44. Young GB, Bolton CF, Austin TW, Archibald YM, Gonder J, Wells GA (1990) The encephalopathy associated with septic illness. Clin Invest Med 13:297-304

45. Zauner C, Gendo A, Kramer L, Funk GC, Bauer E, Schenk P, Ratheiser K, Madl C (2002) Impaired subcortical and cortical sensory evoked potential pathways in septic patients. Crit Care Med 30:1136-1139 